\title{
Application of Two Unknown Quantities and Three Points Interpolation in the Atmospheric Transmittance of Infrared Radiation
}

\author{
Honghui Sun, Min Li and Jianshe Song \\ Dept.of Physics \\ Xi’an Research Inst.of Hi-Tech,Hongqing Town \\ Xi'an, China \\ e-mail: redlightshh@163.com, li-min@163.com,song-jianshe@163.com
}

\begin{abstract}
The atmospheric transmittance of infrared radiation is the important parameter on the studies of infrared radiation, the Computation of atmospheric transmittance is professional and complicated and it is inconvenient for the application of engineering and technology. In order to gained the atmospheric transmittance of infrared radiation rapidly when the height of infrared apparatus place and the down range distance between infrared apparatus and infrared object are changed, Lagrange's two unknown quantities and three points interpolation is used to computer the interpolations of the atmospheric transmittance of infrared radiation by the MODTRAN software, then the available data of atmospheric transmittance are gained. The experimental results show the interpolation method is effective and valuable in engineering applation.
\end{abstract}

Keywords-component: infrared radiation; atmospheric Transmittance;two unknown quantities and three points interpolation ; interpolation method

\section{INTRODUCTION}

The anything in the nature can radiate infrared radiation as long as its temperature is higher than the absoluteness zero, and the characteristic of infrared radiation is always different for different objects. The radiation characteristic of infrared object is the important foundation for the infrared system to realize the detection and recognition of infrared objects. According to the formula of Planck, the infrared radiation of object is decided by the exterior radiation rate and temperature of infrared object. The exterior radiation rate of object is always definite, and its change by the nature reason is always faint. For the exterior temperature of object, besides in-house configuration and inner caloric source, it is also affected by diversiform circumstance factor $\mathrm{s}$. Especially, the atmospheric transmittance is the very important factor and it immediately affects the exterior temperature of object. Otherwise the distance between the infrared system and the infrared object is often distant and the infrared radiation of object is weakened by the atmosphere in course of the transmission, so the atmospheric transmittance is also taken into account.

Because of the detection of infrared objects, infrared imaging and infrared navigation are related to the character of the atmospheric transmittance of infrared radiation, so the transmittance parameters are important on the studies of infrared radiation and are always studied by scientific workers [1]. The precision of the atmospheric transmittance of infrared radiation is different for the different trade scientific workers, it are currently used to compute the atmospheric transmittance of infrared radiation by the professional LOWTRAN, MODTRAN and HITRAN infrared emulational software. A mass of parameters are required for these professional software and it is difficult and inconvenient to be used for other trade scientific workers [2,3].

In general, the atmospheric environment of the infrared object zone is almost changeless in the short period of time. The infrared detector often detect the infrared object in different height and different down range distance between infrared apparatus and infrared object, in order to recognize the object, so the atmospheric transmittance of infrared radiation of the same atmospheric environment but it is in different height and different down range distance is computed again and again. In order to reduce account and improve operation speed, the Lagrange's two unknown quantities and three points interpolation is used to computer the interpolations of the atmospheric transmittance of infrared radiation on based of the data computed by the MODTRAN software, and it is convenient to get the available data of atmospheric transmittance in different height and different down range distance between infrared apparatus and infrared object.

\section{THE PRINCIPLE OF THE LAGRANGE'S TWO UNKNOWN QUANTITIES AND THREE POINT INTERPOLATION}

It is common knowledge that the interpolation is the estimate method between the data points, and the data points are presented by some concourse field. When the people can not quickly compute the needful middle data points, the interpolation method is the valuable implement.

In the field of numerical value approach and data analysis, many unknown quantities interpolation is the classical disposal arithmetic. The simplest interpolation is one unknown quantity and polynomial interpolation, its theory and method are primarily ripe and its productions are widely used. However many unknown quantities and polynomial interpolation is more difficult than one unknown quantity and polynomial interpolation, because the interpolation field of many unknown quantities involves not only the quantity of interpolation but also the geometry distributing of interpolation. In recent twenty and thirty years, 
the theory and method research of many unknown quantities and polynomial interpolation is quickly developed and the interpolation method is widely used to the computation of polynomial function, the outline design of curved surface and so on.

Presently, the two unknown quantities and three points interpolation is frequently used in the field of engineering and technology, its theory is showed as given below.

It are $n \times m$ points $\left(x_{i}, y_{i}\right)(i=0,1, \cdot \cdots n-1 ; j=0,1, \cdot m-1)$ in the rectangular extent, and its values is $z_{i j}=z\left(x_{i}, y_{j}\right)$. The approximate value $w=z(u, v)$ of the interpolation point $(u, v)$ can be computed by the Lagrange's two unknown quantities and three points interpolation [4].

It is supposed the coordinates of the $n \times m$ points in the rectangular extent are $x_{p}<x_{p+1}<x_{p+2}$ and $y_{q}<y_{q+1}<y_{q+2}$ in the two directions of coordinate axis. Then the approximate value $w=z(u, v)$ of the interpolation point $(u, v)$ can be computed by equation (1) as given below.

$$
z(x, y)=\sum_{i=p}^{p+2} \sum_{j=q}^{q+2}\left(\prod_{\substack{k=p \\ k \neq i}}^{p+2} \frac{x-x_{k}}{x_{i}-x_{k}}\right)\left(\prod_{\substack{l=p \\ l \neq j}}^{q+2} \frac{y-y_{l}}{y_{j}-y_{l}}\right) z_{i j}
$$

Apparently, distributing of the interpolation points is more even and dense and the corresponding function value is more accurate.

According to the arithmetic theory, the author programs the two unknown quantities and three points interpolation by VC 6.0, and the program codes are showed as given below.

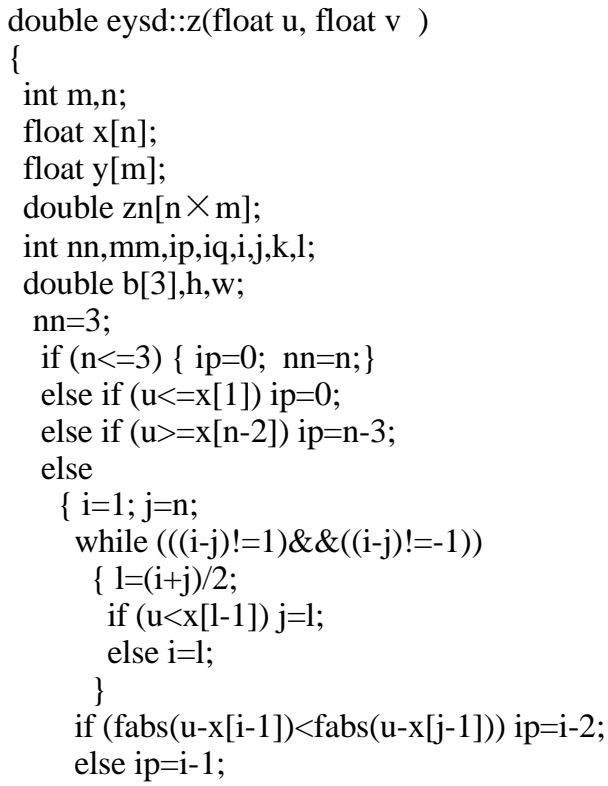

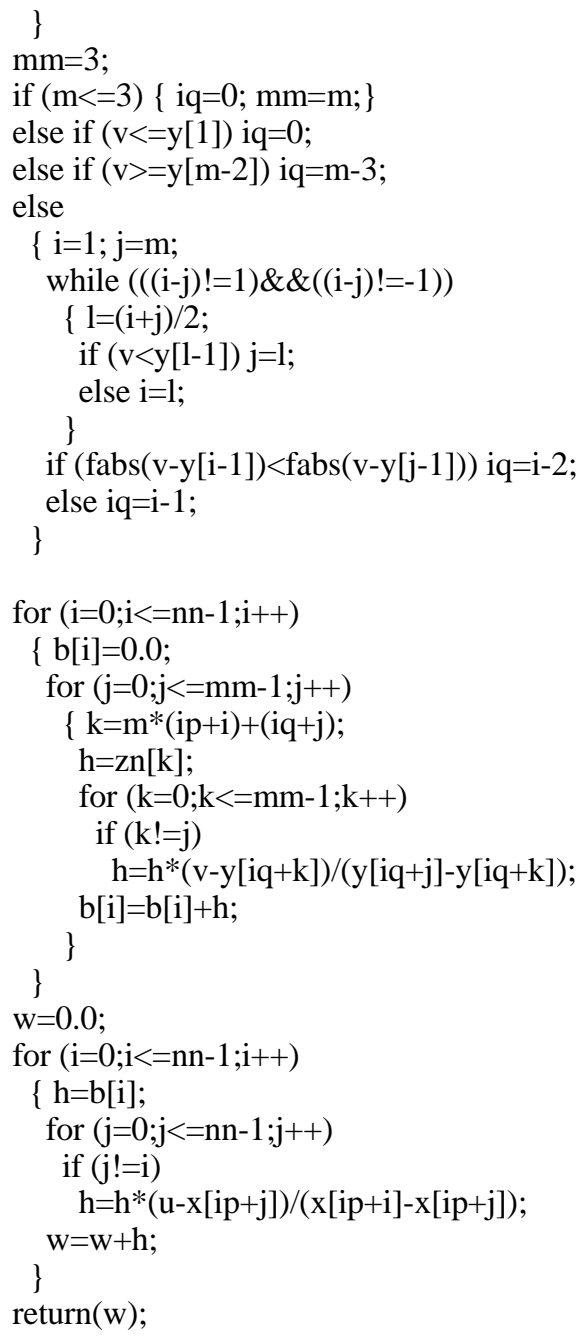

III. The ApPlicAtion OF Two UnKNOWN QUANTITIES AND THREE POINTS INTERPOLATION IN THE ATMOSPHERIC TRANSMITTANCE OF INFRARED RADIATION

In the application field of engineering and technology, the MODTRAN (moderate precision) infrared emulational software is often used to compute the atmospheric transmittance of infrared radiation by scientific workers. The atmospheric transmittance graph of infrared radiation of the infrared object zone is shown in Fig.1.The infrared wavenumber is in the extent $\left(2000 \sim 3300 \mathrm{~cm}^{-1}\right)$, namely the infrared wavelength is the extent $(3 \sim 5 \mu \mathrm{m})$. The extent ( $3 \sim$ $5 \mu \mathrm{m}$ ) is in common use and the material InSb and HgCdTe can be used to produce the infrared detector working in the middle infrared wave band. 


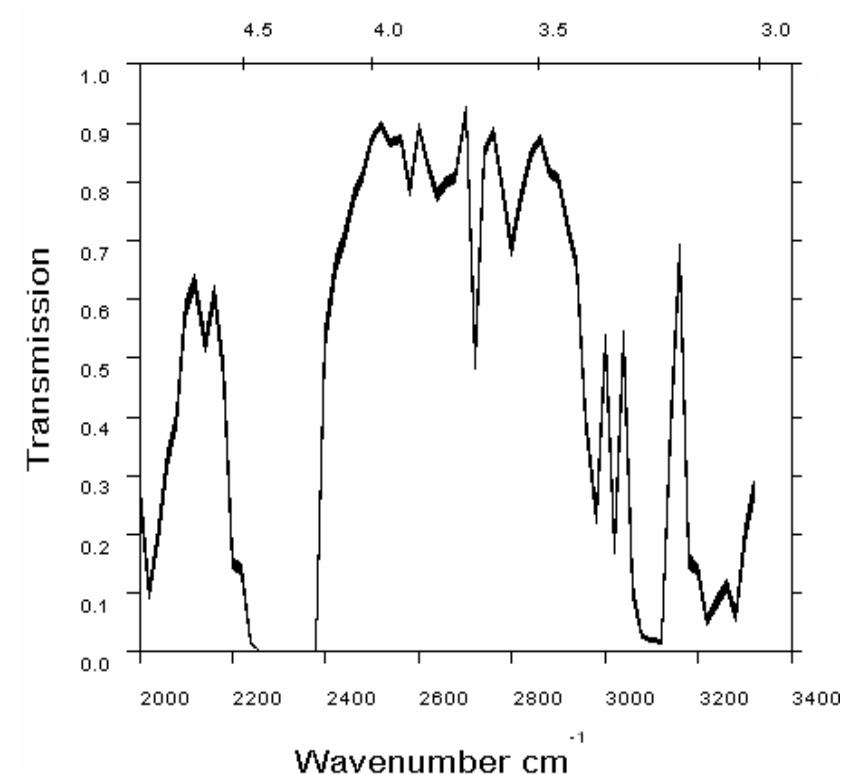

Figure 1. The atmospheric transmittance graph of infrared radiation

In the infrared emulational model, the observer height is $4 \mathrm{~km}$ the down range distance is $9 \mathrm{~km}$, and the object height is $1 \mathrm{~km}$,the model atmosphere is mid latitude summer and execute with multiple scattering, the atmospheric components mainly comprise water vapor, ozone, methane, nitrous oxide, carbon monoxide and the $\mathrm{CO}_{2}$ mixing ratio is 330 ppmv, the aerosol model used is rural zone and the visible spectrometry is $23 \mathrm{~km}$.

The atmospheric transmittance of infrared radiation changes as the observer height changes in the extent (3$8 \mathrm{~km}$ ) and the down range distance changes in the extent (7$14 \mathrm{~km}$ ), and the average atmospheric transmittance data in the same atmospheric environment are shown in Table 1 . In order to get more accurate data, the observer height and the down range distance can be used to be two parameters and be inputted to equation (1), so the approximate value of the interpolation point can be computed by the Lagrange's two unknown quantities and three point interpolation. The data as shown in Table 2 are the interpolation data of the average atmospheric transmittance at interval of $0.25 \mathrm{~km}$, and the observer height changes in the extent (3-4 km ) and the down range distance changes in the extent $(7-9 \mathrm{~km})$, the interpolation method improve the operation speed and efficiency and the error between interpolation data and MODTRAN data is less.

TABLE I. The DATA of the AVERAge AtMospheric TRANSMITTANCE

\begin{tabular}{c|c|c|c|c|c|c}
\hline \multirow{2}{*}{$\begin{array}{c}\text { Down Range } \\
\text { Distance(km) }\end{array}$} & \multicolumn{7}{|c}{ The Observer Height $(\mathbf{k m})$} \\
\cline { 2 - 7 } & $\mathbf{3}$ & $\mathbf{4}$ & $\mathbf{5}$ & $\mathbf{6}$ & $\mathbf{7}$ & $\boldsymbol{8}$ \\
\hline $\mathbf{7}$ & 0.3887 & 0.4248 & 0.4554 & 0.4813 & 0.5035 & 0.5228 \\
\hline $\boldsymbol{8}$ & 0.3656 & 0.4020 & 0.4330 & 0.4594 & 0.4820 & 0.5018 \\
\hline $\mathbf{9}$ & 0.3451 & 0.3817 & 0.4130 & 0.4397 & 0.4626 & 0.4828 \\
\hline $\mathbf{1 0}$ & 0.3268 & 0.3635 & 0.3950 & 0.4218 & 0.4450 & 0.4654 \\
\hline $\mathbf{1 1}$ & 0.3103 & 0.3469 & 0.3785 & 0.4055 & 0.4289 & 0.4495 \\
\hline $\mathbf{1 2}$ & 0.2953 & 0.3319 & 0.3634 & 0.3905 & 0.4140 & 0.4348 \\
\hline $\mathbf{1 3}$ & 0.2815 & 0.3180 & 0.3495 & 0.3766 & 0.4002 & 0.4212 \\
\hline $\mathbf{1 4}$ & 0.2688 & 0.3052 & 0.3366 & 0.3637 & 0.3874 & 0.4084 \\
\hline
\end{tabular}

TABLE II. THE INTERPOLATION DATA OF THE AVERAGE ATMOSPHERIC TRANSMITTANCE

\begin{tabular}{c|c|c|c|c|c}
\hline \multirow{2}{*}{$\begin{array}{c}\text { Down Range } \\
\text { Distance (km) }\end{array}$} & \multicolumn{5}{|c}{ The Observer Height (km) } \\
\cline { 2 - 6 } & $\mathbf{3}$ & 3.25 & 3.5 & 3.75 & 4 \\
\hline 7 & 0.3887 & 0.3982 & 0.4074 & 0.4163 & 0.4248 \\
\hline $\mathbf{7 . 2 5}$ & 0.3826 & 0.3922 & 0.4014 & 0.4103 & 0.4188 \\
\hline $\mathbf{7 . 5}$ & 0.3768 & 0.3864 & 0.3956 & 0.4045 & 0.4131 \\
\hline $\mathbf{7 . 7 5}$ & 0.3711 & 0.3807 & 0.3900 & 0.3989 & 0.4075 \\
\hline $\boldsymbol{8}$ & 0.3656 & 0.3752 & 0.3845 & 0.3934 & 0.4020 \\
\hline $\mathbf{8 . 2 5}$ & 0.3602 & 0.3699 & 0.3791 & 0.3881 & 0.3967 \\
\hline $\mathbf{8 . 5}$ & 0.3551 & 0.3647 & 0.3740 & 0.3830 & 0.3916 \\
\hline $\mathbf{8 . 7 5}$ & 0.3500 & 0.3597 & 0.3690 & 0.3779 & 0.3866 \\
\hline $\boldsymbol{9}$ & 0.3451 & 0.3547 & 0.3641 & 0.3730 & 0.3817 \\
\hline
\end{tabular}

\section{THE CONCLUSION}

The Lagrange's two unknown quantities and three point interpolation is used to compute not only the average atmospheric transmittance of infrared radiation but also the other two-dimensional interpolation. It is apparently that the interpolation method is useful for the scientific workers, and the experimental results show the interpolation method is effective and valuable in engineering application..

\section{REFERENCES}

[1] Wang Xuewei, Li Ke, "Research on Modelind and Simulation Method of Infrared Imaging System,” Computer \& Digital Engineering, vol. 40, pp. 124-126, June 2012.

[2] Lu Yuan ,Ling Yong-shun , "The Simple Method to Calculate the AtmosphereTransmittance of Infrared Radiation”, Infrared Technology, vol .25, pp. 45-49, May 2003.

[3] Zhou Guo-hui, Liu Xiang-wei, Xu ji-wei, "A Math Model of Calculate the Atmospheric Transmittance Of Infrared Radiation ,” Infrared Technology ,vol. 30 , pp. 331-334, une 2008.

[4] Ge Zhong-zhe, "Application of Two Unknown Quantities and Three Points Interpolation in the mechanical part design by the computer," Journal of Ezhou university, vol .17, pp.13-16, February 2010. 